This is an electronic reprint of the original article. This reprint may differ from the original in pagination and typographic detail.

Author(s): Käyhkö, Marko; Palosaari, M. R. J.; Laitinen, Mikko; Arstila, Kai; Maasilta, Ilari; Fowler, J. W.; Doriese, W. B.; Ullom, J. N.; Sajavaara, Timo

Title: $\quad$ Minimum detection limits and applications of proton and helium induced X-ray emission using transition-edge sensor array

Year: $\quad 2017$

Version:

Please cite the original version:

Käyhkö, M., Palosaari, M. R. J., Laitinen, M., Arstila, K., Maasilta, I., Fowler, J. W., Doriese, W. B., Ullom, J. N., \& Sajavaara, T. (2017). Minimum detection limits and applications of proton and helium induced X-ray emission using transition-edge sensor array. Nuclear Instruments and Methods in Physics Research. Section B : Beam Interactions with Materials and Atoms, 406(Part A), 130-134. https://doi.org/10.1016/j.nimb.2017.02.040

All material supplied via JYX is protected by copyright and other intellectual property rights, and duplication or sale of all or part of any of the repository collections is not permitted, except that material may be duplicated by you for your research use or educational purposes in electronic or print form. You must obtain permission for any other use. Electronic or print copies may not be offered, whether for sale or otherwise to anyone who is not an authorised user. 


\title{
MINIMUM DETECTION LIMITS AND APPLICATIONS OF PROTON AND HELIUM INDUCED X-RAY EMISSION USING TRANSITION-EDGE SENSOR ARRAY
}

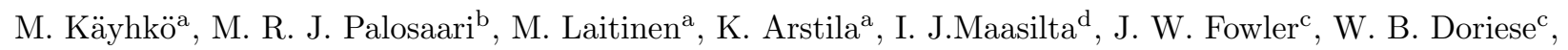 \\ J. N. Ullom ${ }^{\text {c }}$, T. Sajavaara ${ }^{\mathrm{a}}$ \\ ${ }^{a}$ University of Jyvaskyla, Department of Physics, Postal address: P.O. Box 35, Postal code: FI-40014, City (postal office): \\ University of Jyvaskyla, Country: Finland \\ ${ }^{b}$ Stresstech Oy Tikkutehtaantie 1, 40800 Vaajakoski, Finland \\ ${ }^{c}$ National Institute of Standards and Technology, 325 Broadway, Boulder, CO 80305, USA \\ ${ }^{d}$ University of Jyvaskyla, Department of Physics, Nanoscience Center, Postal address: P.O. Box 35, Postal code: FI-40014, City \\ (postal office): University of Jyvaskyla, Country: Finland
}

\begin{abstract}
We have determined minimum detection limits, MDLs, for elements $14 \leq Z \leq 86$ using a transition-edge sensor array, TES array, and as a comparison using an Amptek X-123SDD silicon drift detector, SDD. This was done using a $3 \mathrm{MeV}$ proton beam and a $5.1 \mathrm{MeV}$ helium beam. MDLs were determined for a thin film sample on top of $\mathrm{C}$ substrate, and for a bulk sample containing mostly Al. Due to the smaller peak-to-background ratio, lower detection limits were obtainable using the TES array for most of the elements. However, for elements $30 \leq Z \leq 45$ the performance of the TES array was not as good as the SDD performance. This is due to the limitations of the TES used at energies $>10 \mathrm{keV}$. The greatest advantage of TES comes, however, when detecting low intensity peaks close to high intensity peaks. Such a case was demonstrated by measuring a fly ash with overlapping Ti, $\mathrm{V}$, Ba, and Ce peaks, where minimum detection limits of $\mathrm{V}$, Ba, and Ce were decreased by factor of 620,400 , and 680 , respectively compared to the SDD.
\end{abstract}

Keywords: PIXE, TES, Minimum Detection Limit, Helium-induced X-ray Emission

\section{Introduction}

Particle-induced X-ray emission, PIXE, is a technique capable of detecting concentrations even in parts per million level [1]. Studying small concentration levels is essential in many situations, such as when studying biological samples, atmospheric aerosols, art or archaeological objects [1]. There are many factors limiting the minimum detection limit MDL: the main elemental composition of the sample, peak-tobackground ratio, detection efficiency, etc. [1]

Transition-edge sensor, TES, having wide-energy range and great energy resolution has been demonstrated to be a good tool in X-ray spectroscopy $[2,3]$. Energy resolution of $3.09 \mathrm{eV}$ was previously demonstrated in our setup [4], and even sub eV resolution has been demonstrated by other authors [5] for detectors

Email address: marko.kayhko@jyu.fi (M. Käyhkö) operating at lower energies. Good energy resolution makes it easier to separate X-ray lines close in energy, such as Ti K $\beta(4.9318 \mathrm{keV})$ and V K $\alpha(4.9522 \mathrm{keV})$. This makes the detection of trace elements close to minor or major elements easier than with the commonly used silicon drift detectors, SDDs. On the downside, a single TES pixel is not capable of such high count rates as SDDs, the non-linear energy response adds complexity and each pixel in a TES array detector has its unique behavior.

\section{Experimental methods}

The measurement setup consists of array of 160 TES pixels detailed in Refs. [4, 3]. Pixels are read with a time division multiplexed SQUID read-out [6]. The measurement chamber with the TES array, silicon drift detector, and sample holder as the main components is illustrated in Fig. 1. In all measurements, 


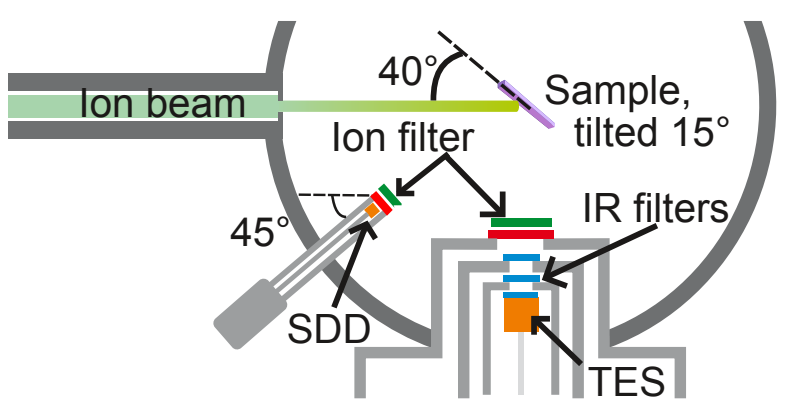

Figure 1: A schematic view of the measurement chamber. The sample is at 40 degree angle with respect to the beam and tilted 15 degree relative to the $2 \mathrm{D}$ measurement plane. Transitionedge sensor array, TES, is at 90 degree angle with respect to the beam and the detector chip is $203 \mathrm{~mm}$ away from the sample. Silicon drift detector, SDD, is at 45 degree angle compared to the beam and the detector crystal is $199 \mathrm{~mm}$ away from the sample. Be filters are used in front of both detectors with $100 \mu \mathrm{m}$ and $125 \mu \mathrm{m}$ thicknesses for SDD, and TES, respectively. Between the sample and TES, there is also 3 infrared filters with $225 \mathrm{~nm}$ thick $\mathrm{Al}$ on $280 \mathrm{~nm}$ thick SiN on each, and AP3.3 vacuum window from Moxtek. Silicon drift detector has $12.5 \mu \mathrm{m}$ thick Be window.

the refrigerator was cooled down to $65 \mathrm{mK}$ with the TES array operated at $100 \mathrm{mK}$. In the analysis, 98102 pixels were used, while 120 pixels were read. The pixels that were rejected either had some problems with the line shape, SQUID locking or the pixels did not produce any pulses.

All measurements were done with the 1.7 MV Pelletron accelerator at the University of Jyväskylä. The proton beam was produced using the Pelletron light ion source PELLIS [7], and a $3.01 \mathrm{MeV}{ }^{1} \mathrm{H}^{+}$beam was used in all proton measurements. The He-beam was produced with Alphatross, and a $5.11 \mathrm{MeV}^{4} \mathrm{He}^{2+}$ beam was used in all He measurements. The beam was focused to the sample using two quadrupoles after the accelerator, and using a collimator with a $2 \mathrm{~mm}$ diameter located before the sample holder. A sample consisting of thin films of $\mathrm{Ti}, \mathrm{Cr}, \mathrm{Cu}$, and $\mathrm{Ge}$ on a Si wafer was measured in the beginning of every measurement day to obtain an energy calibration for the TES array. The details of the measurements are given in Fig. 1 and in Table 1. The target current was not measured, and the accumulated charge was estimated using known reference materials, solid angles, detector efficiencies and X-ray yields. The estimated charges are shown in Table 1.
Table 1: Details of the performed measurements done using 3.01 MeV proton beam and 5.1 MeV He beam. The Au sample is a thin gold foil on carbon substrate manufactured by Institute for Reference Materials and Measurements, IRMM, and the certificated Au thickness was $(52.5 \pm 2.1) \mu \mathrm{g} / \mathrm{cm}^{2}$. The Al sample is manufactured by NIST, and its standard reference material number is SRM $1255 \mathrm{~b}$.

\begin{tabular}{ccccc}
\hline Sample & $\mathrm{Au}, \mathrm{H}$ & $\mathrm{Al}, \mathrm{H}$ & $\mathrm{Au}, \mathrm{He}$ & $\mathrm{Al}, \mathrm{He}$ \\
\hline Time, min & 60 & 54 & 83 & 103 \\
Cnts/pixel/s & 2.97 & 5.62 & 0.46 & 4.16 \\
Good pixels & 100 & 102 & 98 & 101 \\
Beam Q/ $\mu \mathrm{C}$ & $84(4)$ & $40.8(11)$ & $20.5(10)$ & $35.5(10)$ \\
\hline
\end{tabular}

\section{Analysis methods}

\subsection{Pulse-processing}

All measured pulse shapes for the TES were saved and analyzed later offline. For the pulse processing, home made software was used with optimal filtering algorithm that is orthogonal to constants as described in [8]. After optimal filtering, energy calibration for each pixel was obtained using a known calibration sample containing $\mathrm{Si}, \mathrm{Ti}, \mathrm{Cr}, \mathrm{Cu}$, and Ge. The energy calibration is done after every cooling cycle. Due to the complex nature of the superconducting transition, a cubic spline function is fitted for every pixel. This provides a good energy calibration for the fit region, but does not extrapolate very well outside the fit region, however.

Due to small drifts in the baselines, a drift correction was used. This is mainly caused by the slight drift $(10 \mu \mathrm{K})$ in the bath temperature of the refrigerator during long measurements. Here, a new algorithm was used for correcting the baseline drift caused by the drift in the temperature. Traditionally this is determined as

$$
p h_{d c}=p h\left[1+\alpha\left(B_{j}-B_{0}\right)\right],
$$

where $p h$ is the estimated pulse height, $p h_{d c}$ is the corrected pulse height, $B_{0}$ is the baseline level in the beginning of the measurement, $B_{j}$ is the baseline level for the current pulse, and $\alpha$ is a calibration constant for each pixel [9]. The problem we have had with this approach is that when working in a wide energyrange, the correction has not been satisfactory. The new algorithm is based on the energy calibration of the detector. Let's define a energy calibration function cal so that $E=\operatorname{cal}(p h)$, where $E$ is the energy of the photon hitting the detector. The drift corrected energy is calculated as

$$
E_{d c}=\operatorname{cal}(p h+\alpha \cdot \Delta)-\operatorname{cal}(\alpha \cdot \Delta),
$$


where $\Delta=B_{j}-B_{0}$, and $\alpha$ is a constant calibrated separately for each pixel. When the baseline changes by $\Delta$, then in the energy calibration curve we move from the point $\Delta$ to $p h+\Delta$, instead of just moving from 0 to $p h$. When thinking about this curve in its physical meaning, the pulse height is related to the temperature of the detector. Since a pulse with height $\Delta$ and the constant drift of $\Delta$ in the base level do not have the same thermal response, a correction term $\alpha$ is used. In practice, it is often found that the energy calibration curve using standard cubic spline fitting can behave badly close to the zero energy. Thus, two calibration curves are used, one with a cubic spline fitting for higher energies (term $\operatorname{cal}(p h+\alpha \cdot \Delta)$, and another, 2nd order polynomial fit for lower energies $($ term $\operatorname{cal}(\alpha \cdot \Delta))$.

Pile-up pulses and other bad pulses are rejected from the analysis. Due to this, a dead time correction is needed in quantitative analysis. The dead time correction is defined by the number of accepted events divided by the number of photons arrived at the detector $\chi=N_{\text {good }} / N_{p h}$. The number of photons arrived at the detector can be approximated with the equation

$$
N_{p h} \approx \frac{N_{e v}}{1-\tau \cdot N_{e v} / T}
$$

where $N_{p h}$ is the actual number of photons arrived at the detector, $N_{e v}$ is the number of events (some with pile-up) in the saved data, $\tau$ is the recorded pulse length (16.384 $\mathrm{ms}$ in all measurements), and $T$ is the measurement time as shown in Table 1. The obtained corrections for the proton beam measurements were 0.820 for the thin film sample, and 0.827 for the bulk sample. The corrections for helium beam measurements were 0.949 for the thin film sample, and 0.848 for the bulk sample.

\subsection{Minimum detection limit calculations}

We use the common definition for minimum detection limit yield $Y_{M D L}$

$$
Y_{M D L}=3 \sqrt{B g_{f w h m}}
$$

where $B g_{f w h m}$ is the background area in the full width at half maximum region [1]. This definition is valid in the situation where the peak height is much higher than the background level and the peaks are well separated [10]. The X-ray yield for a thin target can be written as

$$
Y_{M D L}=Y_{i t}(Z) \cdot m_{a, M D L} \cdot \gamma(Z),
$$

where $m_{a, M D L}$ is the concentration (in $\left.\mathrm{g} / \mathrm{cm}^{2}\right), Y_{i t}(Z)$ is theoretical yield in unit $1 / \mathrm{str} \cdot \mathrm{Cg} / \mathrm{cm}^{2}$ containing cross sections and known physical constants, and $\gamma$ is the experimental yield (in unit $\mathrm{C} \cdot \mathrm{str}$ ) calibrated using standards, containing experimental parameters like solid angle, filter transmission, and detector efficiency [1]. Now we can write

$$
m_{a, M D L}=\frac{3 \sqrt{B g_{f w h m}}}{Y_{i t}\left(Z, E_{0}\right) \gamma(Z)} .
$$

The same equation holds for a thick target specimen, with the exception that the theoretical yield is in unit $1 /$ str.C.ppm [1].

The extraction of peak areas for the TES spectra were done using fityk version 1.3.0 [11]. The peaks were fitted using Voigt profiles. The X-ray background was fitted using a self-made computer code, the X-ray yields were calculated using GUPIXwin version 2.2.4 [12] and SDD spectra were analyzed using GUPIXwin, except for the background determination which was fitted using the self-made code.

\subsubsection{TES area corrections \\ $K$ shell}

$\mathrm{K} \alpha$ transition is split into two lines: $\mathrm{K} \alpha_{1}$, and $\mathrm{K} \alpha_{2}$ and the ratio is circa 100/50 [13]. In minimum detection calculations it is thus always beneficial to include both of these lines in the calculation. When one includes $\mathrm{K} \alpha_{2}$ in the calculation, the increase in area is $50 \%$, and the increase in required area for the detection limit (due to the increased background area) of an element increases in the worst case by $\sqrt{2} \approx 41 \%$. The width of the analysis area is then defined as

$$
W=F W H M+\min \left(\Delta E_{K \alpha 1-K \alpha 2}, F W H M\right),
$$

where $F W H M$ is the full width at half maximum, and $\Delta E_{K \alpha 1-K \alpha 2}$ is the energy difference of the $K \alpha_{1}$, and $K \alpha_{2}$ lines. In other words this means the energy region spans from $E_{K \alpha 2}-H W H M$ to $E_{K \alpha 1}+H W H M$, when $\Delta E_{K \alpha 1-K \alpha 2}<F W H M$, where $H W H M$ is the half width at half maximum. When $\Delta E_{K \alpha 1-K \alpha 2}>$ $F W H M$, we analyze the FWHM region around both $K \alpha_{1}$, and $K \alpha_{2}$ peaks. Since the integration area for different elements is now different relative to the full intensities, we use a correction term $A$. The correction term is defined so that completely separated $\mathrm{K} \alpha_{1}$, and $\mathrm{K} \alpha_{2}$ peaks have $A=1$. Assuming Gaussian line shapes we get

$$
A=\frac{\left[\operatorname{erf}(\sqrt{\ln 2})+\operatorname{erf}\left(\frac{\Delta^{\prime}+\sqrt{2 \ln 2}}{\sqrt{2}}\right)\right]}{2 \cdot \operatorname{erf}(\sqrt{\ln 2})},
$$


when $\Delta^{\prime}=\frac{\left(E_{K \alpha_{1}}-E_{K \alpha_{2}}\right)}{\sigma}<2 \sqrt{2 \ln 2}$. When we have $\Delta^{\prime}=\frac{\left(E_{K \alpha_{1}}-E_{K \alpha_{2}}\right)}{\sigma}>2 \sqrt{2 \ln 2}$ we obtain

$$
A=1+\frac{\left[\operatorname{erf}\left(\frac{\Delta^{\prime}+\sqrt{2 \ln 2}}{\sqrt{2}}\right)+\operatorname{erf}\left(-\frac{\Delta^{\prime}+\sqrt{2 \ln 2}}{\sqrt{2}}\right)\right]}{2 \cdot \operatorname{erf}(\sqrt{\ln 2})} .9
$$

\section{$L$ shell}

$\mathrm{L} \alpha$ is split into two lines: $\mathrm{L} \alpha_{1}$, and $\mathrm{L} \alpha_{2}$ and the ratio is circa 100/11 [13]. Thus in minimum detection calculations we want to use only the $L \alpha_{1}$ line. But since for lighter elements these lines overlap, we will include an area correction term like we did for the $\mathrm{K}$ shell. The case where $L \alpha_{1}$, and $L \alpha_{2}$ are completely separated has a correction $A=1$. The obtained correction is now

$$
A=\frac{\operatorname{erf}(\sqrt{\ln 2})+\frac{1}{18}\left[\operatorname{erf}\left(H_{3}(\delta)\right)-\operatorname{erf}\left(H_{2}(\delta)\right)\right]}{\operatorname{erf}(\sqrt{\ln 2})},
$$

where $H_{3}=\frac{\sqrt{2 \ln 2}+\delta}{\sqrt{2}}$, and $H_{2}=\frac{-\sqrt{2 \ln 2}+\delta}{\sqrt{2}}$, and $\delta=$ $\frac{\left(E_{L \alpha 1}-E_{L \alpha 2}\right) \cdot 2 \sqrt{2 \ln 2}}{\text { FWHM }}$.

\subsubsection{Multiple ionization corrections}

For a $5.1 \mathrm{MeV}$ He beam, the multiple ionization $[14,15]$ becomes a notable effect and requires corrections for the TES analysis. Since the majority of the observed $\mathrm{x}$-rays are from single ionization transitions, the used correction term is the $\mathrm{KL}^{0}$ fraction, i.e. the fraction of photons from single ionization. This was determined for the calibration sample, and it was $0.55(2), 0.76(8), 0.81(3), 0.83(3)$, for $\mathrm{Si}, \mathrm{Ti}$, $\mathrm{Cr}$, and $\mathrm{Cu}$, respectively. For this data, we fitted a phenomenological function $f(Z)$ for the $\mathrm{KL}^{0}$ fraction obtaining

$$
f(Z)=1-a_{0} \cdot Z^{-a_{1}}
$$

with $a_{0}=21.033$ and $a_{1}=1.453566$.

The multiple ionization correction for the L-shell could not be estimated due to the lack of L $\alpha$ transitions in the measured spectra. The multiple ionization of $\mathrm{L} \alpha$ has been studied little [16], and we could not find previous studies that would have given the answer for this.

\subsubsection{Full widths at half maximum}

In the contex of minimum detection limit determination, with full width at half maximum (FWHM) we
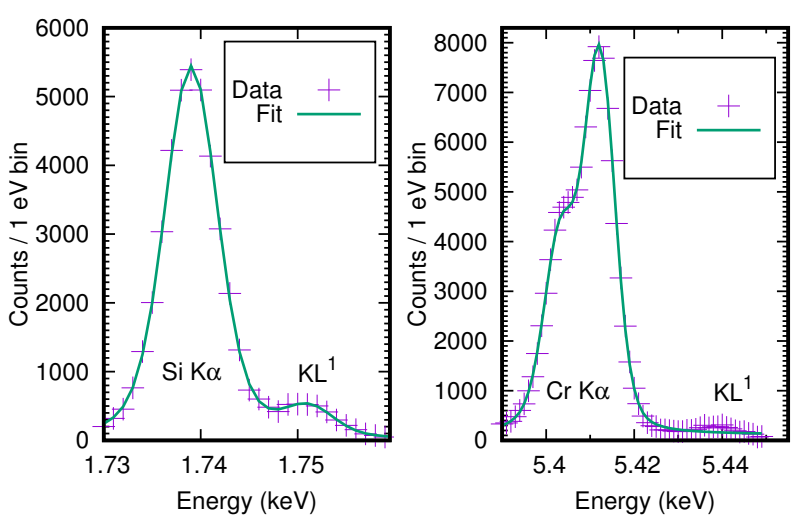

Figure 2: Fitted $\mathrm{Si} \mathrm{K} \alpha$ and $\mathrm{Cr} \mathrm{K} \alpha$ peaks with fitted FWHMs being $(6.36 \pm 0.03) \mathrm{eV}$, and $(6.90 \pm 0.06) \mathrm{eV}$, respectively. For the Si K $\alpha$ complex, two Voigt profiles were used $\left(\alpha_{1}\right.$, and $\left.\alpha_{2}\right)$ and for the $\mathrm{Cr} \mathrm{K} \alpha$ complex seven Voigt profiles were used: 5 for $\alpha_{1}$ and 2 for $\alpha_{2}$ [18]. Both spectra were measured using a $3.01 \mathrm{MeV}$ proton beam.

mean the convolution of instrumental FWHM, natural line widths [17], and X-ray fine structure $\left(\alpha_{1}\right.$, and $\left.\alpha_{2}\right)$. The instrumental resolution was determined from the proton measurements for $\mathrm{Si} \mathrm{K} \alpha, \mathrm{Al} \mathrm{K} \alpha$, and $\mathrm{Cr} \mathrm{K} \alpha$. Si, and $\mathrm{Al}$ peaks were fitted with two Voigt profiles $\left(\mathrm{K} \alpha_{1}\right.$ and $\left.\mathrm{K} \alpha_{2}\right)$, whereas $\mathrm{Cr} \mathrm{K} \alpha$ complex was fitted with 7 peaks (5 $\mathrm{K} \alpha_{1}$ and $2 \mathrm{~K} \alpha_{2}$ peaks) [18]. The obtained instrumental energy resolutions were: $(6.59 \pm 0.09) \mathrm{eV}(\mathrm{Si} \mathrm{K} \alpha),(6.90 \pm 0.06) \mathrm{eV}(\mathrm{Cr} \mathrm{K} \alpha)$ in the first measurement day, and $(6.45 \pm 0.07) \mathrm{eV}(\mathrm{Al}$ $\mathrm{K} \alpha),(6.36 \pm 0.03) \mathrm{eV}(\mathrm{Si} \mathrm{K} \alpha)$ in the second measurement day. In the analysis, we used the average value $6.6 \mathrm{eV}$ for the instrumental resolution. An example of fitted Si K $\alpha$, and $\mathrm{Cr} \mathrm{K} \alpha$ peaks is shown in Fig. 2.

For SDD the FWHM was estimated by GUPIXwin, where it was fitted using the equation $F W H M=$ $2.355 / A 2 \sqrt{A 4+A 5 \cdot E}$, where $A 2=143.6741 / \mathrm{keV}$, $A 4=33.1219$, and $A 5=9.65941 / \mathrm{keV}$ are calibration constants.

\section{Results and Discussion}

The minimum detection limits for both samples are plotted in Figs. 3, and 4. The shape of the minimum detection limit curve is mainly influenced by the $\mathrm{x}$ ray yield and the shape of the background. Both $\mathrm{x}$ ray yield and background decrease with increasing $Z$. At first the nearly exponential background decrease (see Fig. 5) dominates and improves the minimum detection limits until at some point the background is near zero and $\mathrm{x}$-ray yield starts to dominate and worsens the detection limits. Thus two "U-shapes" 
are formed, one for the K-shell and the another for the L-shell.

A comparison of proton and helium backgrounds for SRM 1255b sample (bulk Al) is show in Fig. 5. The background from the helium measurement is lower than for the proton beam, which allows lower detection limits with He beam in some cases.

We measured trace elements in a fly ash sample with 50:50 ratio of bio and peat fuel. The full spectrum is shown in Fig. 6 and zoomed in $4.2-5.7 \mathrm{keV}$ region is shown in Fig. 7. The overlapping of Ti K lines, V K lines, Ba L lines, and Ce $\mathrm{L}$ lines is evident in Fig. 7. In the case of overlapping peaks, the equation (4) does not hold [10]. We follow the definitions in [10]: If peak separation, sep, $>2$ FWHM peaks are separated, if sep $<\mathrm{FWHM} / 10$ the overlap is severe, otherwise there is some overlap. For the $\alpha_{1}$ peaks of the previously mentioned elements, this means that for Ti, V, and Ba there is no overlap, and for Ce there is some overlap in the TES spectrum. For SDD, there is some overlap for $\mathrm{Ti}, \mathrm{V}, \mathrm{Ba}$ and severe overlap for Ce. The MDL in these situations can be written as

$$
M D L=\text { cons } \cdot F W H M^{n},
$$

where $n=0.5$ for non overlapping peaks, $n=1.5$ for severe overlap of two peaks, and $n=0.5-1.5$ when there is some overlapping [10]. This equation assumes that the peak areas are roughly the same, and that the background level is high relative to the peaks. Using resolutions of $6.6 \mathrm{eV}$ for the TES and $145 \mathrm{eV}$ for the SDD, and assuming the same detection geometry and efficiency, we can calculate that the MDL improves by a factor of 620 ( $n=1.4825$ for SDD), $400(n=$ 1.3947 for SDD), and $680(n=0.5$ for TES) for $\mathrm{V}$, $\mathrm{Ba}$, and Ce, respectively. The $n$ values for peaks with some overlap were approximated linearly using values $n=0.5$ at $\operatorname{sep}=2 \cdot F W H M$, and $n=1.5$ at $\operatorname{sep}=$ $0.1 \cdot F W H M$.

\section{Conclusions}

We have observed improvements in the detection limits for the TES array compared to the SDD detector for the most elements in the case of well separated peaks. Elements $30 \leq Z \leq 45$ are the hardest to detect because of the limitations in detection efficiency for high energy X-rays with our type of TES and high absorption for low energy X-rays in the current setup. When comparing the helium and the proton beam, the helium beam is better for thin film samples whereas the proton beam is better for bulk samples due to the deeper penetration depth.

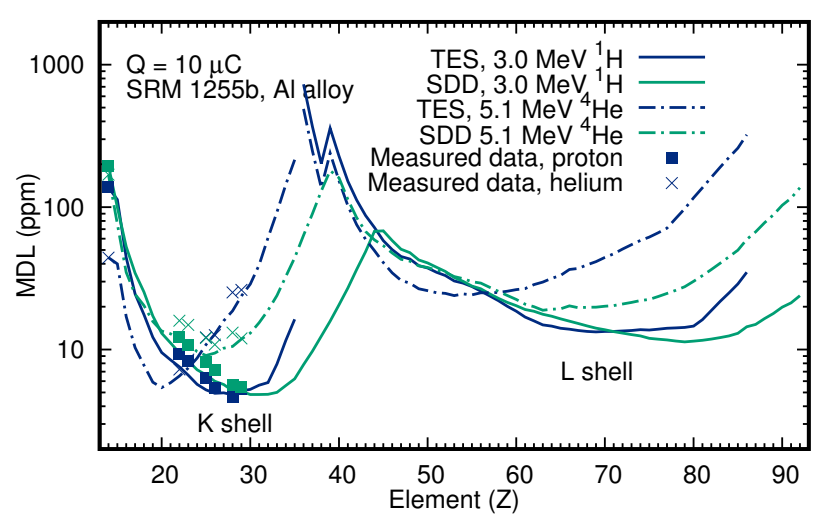

Figure 3: Minimum detection limits for elements $Z \geq 14$ using $\mathrm{K} \alpha$ and $\mathrm{L} \alpha$ peaks in the analysis (both shown for SDD) for elements in matrix made mostly of $\mathrm{Al}$ (SRM 1255b). The odd behaviour in MDL for TES at $Z=38$ is due to the Bi Mshell absorption edge ( $\mathrm{Bi}$ is the absorber material in the TES detector). At the peak positions $(Z \approx 40)$, the L-shell detection limits start to be lower than the K-shell detection limits.

The advantage of TES becomes clear when detecting overlapping peaks of trace and minor elements. In this case, the minimum detection limit scales as $F W H M^{n}$, where $0 \leq n \leq 1.5$ for two identical peaks. Such an example is the measured fly ash sample with overlapping Ti, V, Ba, and Ce peaks at around $5 \mathrm{keV}$ energy. In this case, there was an overlap of Ti, V, Ba, and Ce lines. An improvement in the detection limit of 620,400 , and 680 was calculated for V, Ba, and Ce, respectively for the measured fly ash. In this analysis, however, there were slightly different experimental parameter for the detectors: different detection angles, different X-ray filters (ion filter in Fig. 1), and different vacuum windows. These effects were not taken into account in the normalization. Also the different detection angles lead to slightly different secondary electron Bremsstrahlung background, which also affects the detection limits.

\section{Acknowledgements}

This work was partly supported by the Academy of Finland Centre of Excellence in Nuclear and Accelerator Based Physics (Ref. 251353). We thank Jyväskylän Energia Oy for providing the fly ash sample. 


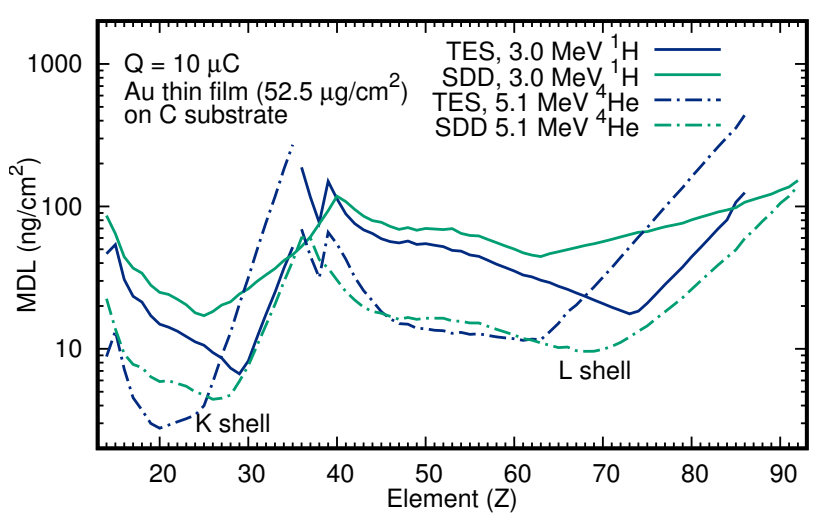

Figure 4: Minimum detection limits for elements $Z \geq 14$ using $\mathrm{K} \alpha$ and $\mathrm{L} \alpha$ peaks in the analysis (both shown for SDD) for thin gold film on carbon substrate. The odd behaviour in MDL for TES at $Z=38$ is due to the Bi M-shell absorption edge. At the peak positions $(Z \approx 40)$, the L-shell detection limits start to be lower than the K-shell detection limits.

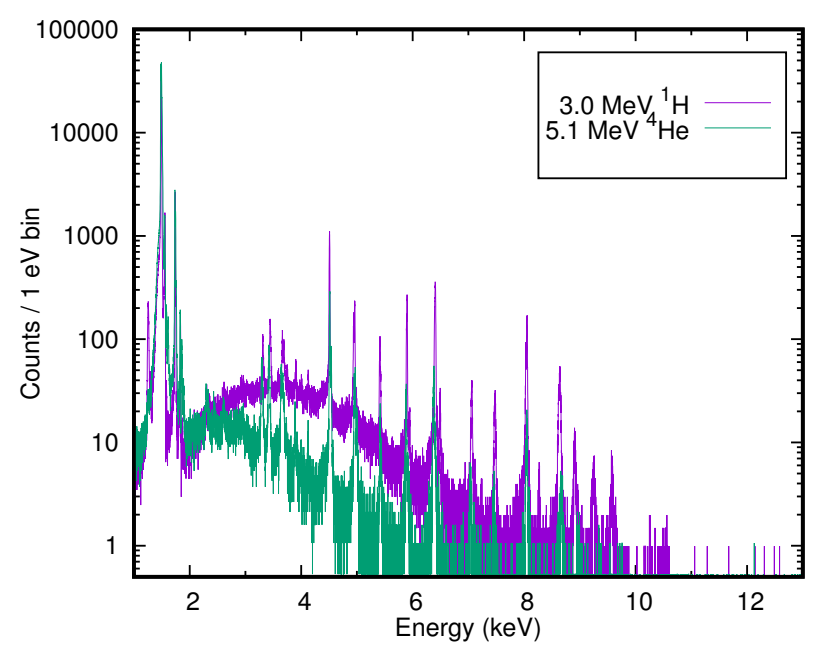

Figure 5: Background comparison of the He and proton beams for the TES detector with $3.0 \mathrm{MeV}$ proton and 5.1 MeV He beams for the bulk Al sample. Both spectra are normalized to $Q=10$ particle $\cdot \mu \mathrm{C}$.

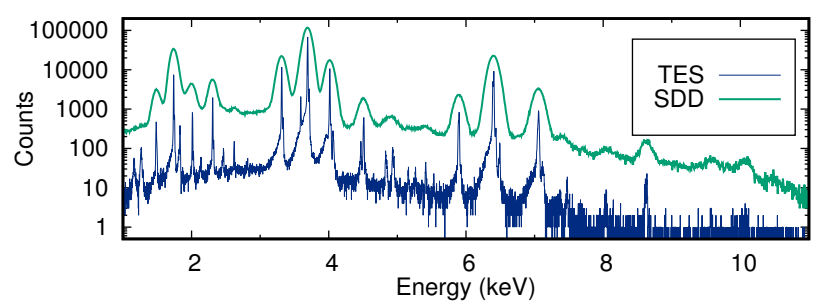

Figure 6: Measured fly ash spectra for SDD and TES. Measured fly ash contained bio / peat fuel with 50:50 ratio.

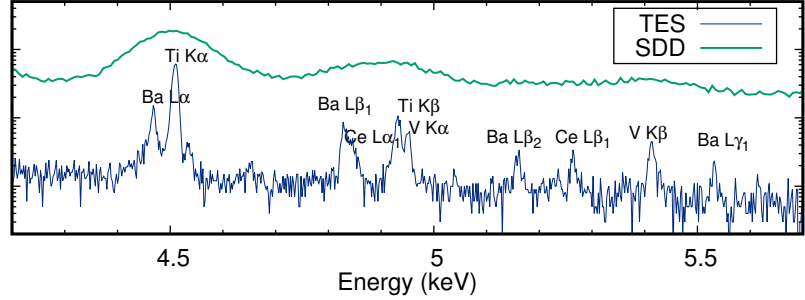

Figure 7: Measured fly ash spectra for SDD and TES, zoomed.

\section{References}

[1] Sven A. E. Johansson, John L. Campbell, and Klas G. Malmqvist. Particle-Induced X-Ray Emission Spectrometry (PIXE). A Wiley-Interscience Publication, New York, 1995.

[2] G.C. Hilton, D.A. Wollman, K.D. Irwin, L. L. Dulcie, N. F. Bergren, and J.M. Martinis. Superconducting transition-edge microcalorimeters for x-ray microanalysis. IEEE Transactions on Applied Superconductivity, 9(2):3177-3181, 1999.

[3] M. R. J. Palosaari, M. Käyhkö, K. M. Kinnunen, M. Laitinen, J. Julin, J. Malm, T. Sajavaara, W. B. Doriese, J. Fowler, C. Reintsema, D. Swetz, D. Schmidt, J. Ullom, and I. J. Maasilta. Broadband ultrahigh resolution spectroscopy of particle induced x-rays: Extending the limits of non-destructive analysis. Physical Review Applied, accepted.

[4] M. R. J. Palosaari, K. M. Kinnunen, J. Julin, M. Laitinen, M. Napari, T. Sajavaara, W. B. Doriese, J. Fowler, C. Reintsema, D. Swetz, D. Schmidt, J. Ullom, and I. J. Maasilta. Transition-Edge Sensors for Particle Induced Xray Emission Measurements. Journal of Low Temperature Physics, 176(3-4):285-290, 2013.

[5] S. J. Lee, J. S. Adams, S. R. Bandler, J. A. Chervenak, M. E. Eckart, F. M. Finkbeiner, R. L. Kelley, C. A. Kilbourne, F. S. Porter, J. E. Sadleir, S. J. Smith, and E. J. Wassell. Fine pitch transition-edge sensor X-ray microcalorimeters with sub-eV energy resolution at $1.5 \mathrm{keV}$. Applied Physics Letters, 107(22):223503, 2015.

[6] W. B. Doriese, J. A. Beall, W. D. Duncan S. Deiker, L. Ferreira, G. C. Hilton, K. D. Irwin, C. D. Reintsema, J. N. Ullom, L. R. Vale, and Y. Xu. Time-division multiplexing of high-resolution x-ray microcalorimeters: Four pixels and beyond. Appl. Phys. Lett., 85:4762, 2004.

[7] T. Kalvas, O. Tarvainen, J. Komppula, M. Laitinen, T. Sajavaara, H. Koivisto, A. Jokinen, and M. P. Dehnel. Recent negative ion source activity at JYFL. In AIP Conference Proceedings, volume 1515, pages 349-358. AIP Publishing, 2013.

[8] B. K. Alpert, R. D. Horansky, D. A. Bennett, W. B. Doriese, J. W. Fowler, A. S. Hoover, M. W. Rabin, and J. N. Ullom. Filters for High Rate Pulse Processing. Journal of Low Temperature Physics, 184:374-381, 2016.

[9] J. W. Fowler, B. K. Alpert, W. B. Doriese, Y.-I. Joe, G. C. O'Neil, J. N. Ullom, and D. S. Swetz. The Practice of Pulse Processing. Journal of Low Temperature Physics, 184:374-381, 2016.

[10] P. J. Statham. Quantifying Benefits of Resolution and Count Rate in EDX Microanalysis. In David B. Williams, Joseph I. Goldstein, and Dale E. Newbury, editors, X-Ray Spectrometry in Electron Beam Instruments, pages 101- 
126. Springer US, 1995. DOI: 10.1007/978-1-4615-1825$9 \_8$.

[11] Marcin Wojdyr. Fityk : a general-purpose peak fitting program. Journal of Applied Crystallography, 43(5):1126$1128,2010$.

[12] J. L. Campbell, N. I. Boyd, N. Grassi, P. Bonnick, and J. A. Maxwell. The Guelph PIXE software package IV. Nuclear Instruments and Methods in Physics Research Section B: Beam Interactions with Materials and Atoms, 268(20):3356-3363, 2010.

[13] A. C. Thompson. X-ray Data Booklet. Lawrence Berkeley National Laboratory, University of California, 2009.

[14] P. G. Burkhalter, A. R. Knudson, D. J. Nagel, and K. L. Dunning. Chemical Effects on Ion-Excited Aluminum $K$ X-Ray Spectra. Physical Review A, 6(6):2093-2101, 1972.

[15] P. Richard, J. Bolger, D. K. Olsen, and C. F. Moore. Molecular structure effects of aluminum $K \beta$ x-ray spectra produced in proton and alpha particle excitation. Physics Letters A, 41(3):269-271, 1972.

[16] T. Hanada, M. Mogi, Kawai Jun, K. Maeda, Y. Sasa, and M. Uda. Nickel L $\alpha$ spectra measured by a high resolution particle induced X-ray emission (PIXE) spectrometer. Nuclear Instruments and Methods in Physics Research Section B: Beam Interactions with Materials and Atoms, 75(1):35-37, 1993.

[17] M. O. Krause and J. H. Oliver. Natural widths of atomic k and 1 levels, ka x-ray lines and several kll auger lines. Journal of Physical and Chemical Reference Data, 8(2):329338, 1979.

[18] G. Hölzer, M. Fritsch, M. Deutsch, J. Härtwig, and E. Förster. $K \alpha_{1,2}$ and $K \beta_{1,3}$ x-ray emission lines of the 3d transition metals. Physical Review A, 56(6):4554-4568, 1997. 